Research, Society and Development, v. 9, n. 8, e125985455, 2020

(CC BY 4.0) | ISSN 2525-3409 | DOI: http://dx.doi.org/10.33448/rsd-v9i8.5455

Extratos de Cedrela fissilis, Melia azedarach e Azadirachta indica em

\title{
Polyphagotarsonemus latus
}

Cedrela fissilis, Melia azedarach and Azadirachta indica extracts on Polyphagotarsonemus latus

\section{Los extractos de Cedrela fissilis, Melia azedarach y Azadirachta indica en Polyphagotarsonemus latus}

Recebido: 09/06/2020 | Revisado: 10/06/2020 | Aceito: 12/06/2020 | Publicado: 27/06/2020

Abimael Gomes da Silva

ORCID: http://orcid.org/0000-0001-7146-9226 Universidade Estadual Paulista, Faculdade de Engenharia, Ilha Solteira, Brasil E-mail:ag.silva@unesp.br Gustavo Haralampidou da Costa Vieira ORCID: http://orcid.org/0000-0001-6879-8424 Universidade Estadual de Mato Grosso do Sul, Cassilândia, Brasil E-mail: gcv@uems.br Thalia Mayara Pereira de Melo ORCID: http://orcid.org/0000-0001-6879-8424 Universidade Estadual de Mato Grosso do Sul, Cassilândia, Brasil E-mail: tha_.melo@hotmail.com

Glaucia Amorim Faria ORCID: http://orcid.org/0000-0003-2474-4840 Universidade Estadual Paulista, Faculdade de Engenharia, Ilha Solteira, Brasil E-mail: glaucia.a.faria@unesp.br

Eliamara Marques da Silva ORCID: http://orcid.org/0000-0002-4152-2046 Universidade Estadual Paulista, Faculdade de Ciências Agrárias e Veterinárias, Brasil E-mail: eliamaramarques@outlook.com

\section{Resumo}

O ácaro-branco Polyphagotarsonemus latus acomete brotações e folhas jovens da seringueira, podendo comprometer a produção e o seu cultivo. Desse modo, objetivou-se determinar o efeito de extratos de diferentes fontes vegetais (Cedrela fissilis, Melia azedarach e 
Azadirachta indica) sobre a mortalidade do ácaro branco Polyphagotarsonemus latus. Foi utilizado o delineamento experimental inteiramente casualizado e os extrato de Cedrela fissilis, Melia azedarach e Azadirachta indica e suas concentrações (0, 5, 10, 20 e 40\%) constituíram os tratamentos. Os extratos foram obtidos de folhas frescas das espécies vegetais, higienizadas, secas e trituradas para obtenção do pó vegetal. Esses, foram diluídos na proporção de 10, 20, 40 e 80 g/100ml de álcool etílico, para cada planta. Após 24 h em repouso os materiais foram filtrados e o resíduo misturado a água deionizada de modo a completar o volume de $200 \mathrm{ml}$. Em placas de Petri contendo uma camada de algodão umedecido, foram depositados discos foliares de folhas de seringueira, com os ácaros. Posteriormente, houve a pulverização sobre os discos. Após 24, 48 e 72 horas foram avaliados a mortalidade dos ácaros. Os dados foram submetidos à análise de variância. Foram utilizados os testes de médias Skott-Knott e análise de regressão para comparar os dados. Todos os extratos apresentam efeito no aumento da mortalidade do ácaro. Os períodos de 48 e 72 horas foram mais expressivos e, para a exposição de 72 horas, é recomendado 28,39\% de qualquer um dos três extratos vegetais.

Palavras-chave: Controle alternativo; Seringueira; Acaros; Acaricida natural.

\section{Abstract}

The white mite Polyphagotarsonemus latus affects shoots and young leaves of the rubber tree, which may compromise production and cultivation. Thus, the objective was to determine the effect of extracts from different plant sources (Cedrela fissilis, Melia azedarach and Azadirachta indica) on the mortality of the white mite Polyphagotarsonemus latus. A completely randomized design was used and the extracts of Cedrela fissilis, Melia azedarach and Azadirachta indica and their concentrations (0, 5, 10, 20 and 40\%) constituted the treatments. The extracts were obtained from fresh leaves of vegetable species, cleaned, dried and crushed to obtain vegetable powder. These were diluted in the proportion of 10, 20, 40 and $80 \mathrm{~g} / 100 \mathrm{ml}$ of ethyl alcohol, for each plant. After $24 \mathrm{~h}$ at rest, the materials were filtered and the residue mixed with deionized water in order to complete the volume of $200 \mathrm{ml}$. In Petri dishes containing a layer of moistened cotton, leaf discs of rubber tree leaves were deposited with the mites. Subsequently, there was spraying on the disks. After 24, 48 and 72 hours, mite mortality was evaluated. The data were submitted to analysis of variance. SkottKnott means tests and regression analysis were used to compare the data. All extracts have an effect on increasing mite mortality. The periods of 48 and 72 hours were more expressive and, for the exposure of 72 hours, $28.39 \%$ of any of the three plant extracts is recommended. 
Keywords: Alternative control; rubber tree; Mites; Natural acaricide.

\section{Resumen}

El ácaro blanco Polyphagotarsonemus latus afecta los brotes y las hojas jóvenes del árbol del goma, lo que puede comprometer la producción y el cultivo. Por lo tanto, el objetivo fue determinar el efecto de los extractos de diferentes fuentes vegetales (Cedrela fissilis, Melia azedarach y Azadirachta indica) sobre la mortalidad del ácaro blanco Polyphagotarsonemus latus. Se utilizó un diseño completamente al azar y los extractos de Cedrela fissilis, Melia azedarach y Azadirachta indica y sus concentraciones (0, 5, 10, 20 y 40\%) constituyeron los tratamientos. Los extractos se obtuvieron de hojas frescas de especies vegetales, se limpiaron, secaron y trituraron para obtener polvo vegetal. Estos se diluyeron en la proporción de 10, 20, 40 y 80 g / $100 \mathrm{ml}$ de alcohol etílico, para cada planta. Después de $24 \mathrm{~h}$ en reposo, los materiales se filtraron y el residuo se mezcló con agua desionizada para completar el volumen de $200 \mathrm{ml}$. En las placas de Petri que contenían una capa de algodón humedecido, se depositaron discos de hojas de árboles de caucho con los ácaros. Posteriormente, hubo rociado en los discos. Después de 24, 48 y 72 horas, se evaluó la mortalidad de los ácaros. Los datos fueron sometidos a análisis de varianza. Skott-Knott significa que se utilizaron pruebas y análisis de regresión para comparar los datos. Todos los extractos tienen un efecto en el aumento de la mortalidad de los ácaros. Los períodos de 48 y 72 horas fueron más expresivos y, para la exposición de 72 horas, se recomienda el 28,39\% de cualquiera de los tres extractos de plantas.

Palabras clave: Control alternativo; Árbol de goma; Acaros; Acaricida natural.

\section{Introdução}

A seringueira [Hevea brasiliensis (Willd. ex. Adr. de Juss.) Müell. Arg., Euphorbiaceae] é uma arbórea nativa da floresta amazônica (Chaves, et al., 2018; Lorenzi, 2000) que está presente nos seringais comerciais por ser fonte de látex, polímero utilizado na produção da borracha natural, e por fornecer madeira (Liu, et al., 2015). Além disso, a seringueira é tida como uma espécie relacionada ao sequestro de carbono atmosférico, estocando na parte aérea e radicular das árvores, na serrapilheira e no solo.

Entre os principais problemas ocorrentes na cultura, destaca-se o estabelecimento de populações de ácaros pragas, sendo as espécies Calacarus heveae Feres (Acari: Eriophyidae) e Tenuipalpus heveae Baker (Acari: Tenuipalpidae) as mais abundantes e as que têm 
apresentado os sintomas mais expressivos em seringais comerciais (Moraes \& Flechtmann, 2008). Moraes e Flechtmann (2008) relatam a ocorrência do ácaro branco Polyphagotarsonemus latus (Tarsonemidae) (Banks, 1904) em brotos e folhas jovens de seringueira.

O principal método de controle de ácaros é o uso de acaricidas sintéticos, porém, esses são de baixa seletividade aos inimigos naturais além de causar resistência às pragas, em alguns casos. Sua utilização inadequada pode ocasionar problemas de contaminação do ambiente e intoxicações em agricultores. Dessa maneira, outras formas de manejo de controle de pragas devem ser consideradas (Alvarenga \& Carmo, 2014) tais como o uso de substâncias menos tóxicas, como os extratos e óleos essências de origem vegetal (Pinto, et al., 2018). Estudos com formulados vegetais demonstraram vantagens quanto as moléculas sintéticas, como baixo custo de obtenção, de baixo risco ambiental, além de promoverem uma resistência muito lenta a pragas (Roel, 2001; Halfeld-Vieira, et al., 2016; Yessinou, et al., 2016).

Dentre as plantas com potencial acaricida, destacam-se as pertencentes à família Meliaceae, como o Cedro - Cedrela fissilis Vell, o Cinamomo - Melia azedarach e o Nim Azadirachta indica A. Juss. (Sariosseiri, et al., 2018). C. fissilis possui cedrelone, um limonóide estruturalmente simples, com propriedades reguladoras de crescimento em insetos, além de serem antifúngicas e antibacterianas (Champagne, et al., 1992). Extratos de $M$. Azedarach são utilizados no controle de pragas, como a mosca-branca (Nardo, et al., 1997) e Spodoptera frugiperda (Oliveira, et al., 2007), devido à presença de limonóides e azadiractina, capazes de inibir o crescimento ou a alimentação de insetos. A. indica é indicada no controle de pragas agrícolas (Moraes \& Flechtmann, 2008; Souza, et al., 2017) por possuir azadiractina, substância de ação inseticida e acaricida de baixa toxicidade ao homem (Schmutterer, 1990).

Tendo em vista a importância de criar novas alternativas de controle do ácaro branco da seringueira e o incentivo de ampliar as pesquisas sobre Meliaceas como matéria prima de acaricida com base vegetal, objetivou-se determinar o efeito de extratos de diferentes fontes vegetais (Cedrela fissilis, Melia azedarach e Azadirachta indica) sobre a mortalidade do ácaro branco (Polyphagotarsonemus latus). 


\section{Metodologia}

Os estudos foram conduzidos no Laboratório de Entomologia da UEMS-UUC, Cassilândia/MS durante o período de agosto, de 2017, a junho de 2018. Os ácaros necessários para realização do experimento foram coletados na Unidade Universitária de Cassilândia, em mudas de seringueira (clone RRIM 600) com aproximadamente três meses, que demonstram alta população da praga. Os ácaros foram identificados de acordo com a metodologia de Moraes e Flechtmann (2008).

Após coleta o material vegetal foi colocado para secagem em estufa de circulação forçada de ar $\left(65^{\circ} \mathrm{C}\right.$ durante $\left.48 \mathrm{~h}\right)$. Após secagem, as folhas de Nim, Cinamomo e Cedro foram trituradas em moinho de facas até a obtenção do pó vegetal. Para preparação dos extratos alcoólicos, foram pesados $10 \mathrm{~g}$ do pó de cada planta e acondicionados separadamente em vidros de coloração âmbar, sendo posteriormente adicionados $100 \mathrm{~mL}$ de álcool etílico. Após $24 \mathrm{~h}$ em repouso o material foi filtrado e reservado, o resíduo da filtragem foi misturado a água deionizada, submetido a agitação e realizada nova filtragem, o liquído extraído foi misturado ao reservado e o volume foi completado para $200 \mathrm{~mL}$, obtendo-se dessa forma uma solução hidroalcóolica na concentração de 5\% (Vendramin \& Castiglioni, 2000; Silva, et al., 2018). Os vidros foram fechados, mas sem vedação. Para a obtenção das concentrações de 10 , 20 e $40 \%$, os valores do pó vegetal foram de 20, 40 e 80 g, respectivamente.

O ensaio foi conduzido em laboratório. Utilizou-se placas de Petri de $9 \mathrm{~cm}$ de diâmetro contendo uma camada de algodão umedecido com água destilada, foram depositados discos foliares de seringueira Clone RRIM 600, recortados com vazador de $3 \mathrm{~cm}$ de diâmetro, obtidos de folhas de mudas de seringueira. Posteriormente foi retirado com auxílio de um pincel o excesso de ovos dos discos foliares, deixando apenas 20 espécimes (formas móveis). A aplicação dos tratamentos foi realizada através de um pulverizador manual utilizando uma dose de $2 \mathrm{ml}$, por disco foliar nas placas de Petri (Schuster, et al., 2009; Melo, et al., 2019). As placas foram vedadas com plástico filme, identificadas e armazenadas em estufa de germinação do tipo B.O.D a 26,0 $( \pm 1,0){ }^{\circ} \mathrm{C}$ e UR de $70( \pm 10) \%$. O plástico das placas foram perfurados para permitir a aeração.

O delineamento experimental utilizado foi inteiramente casualizado em esquema fatorial 3 x 5, três extratos (C. Fissilis, M. azedarach e A. indica) e cinco concentrações ( 0 , $5 \%, 10 \%, 20 \%$ e 40\%), com quatro repetições, sendo para cada repetição utilizado 20 exemplares móveis do ácaro. Para quantificar a Mortalidade, foram considerados a larvas e 
adultos mortos no disco e no algodão. Os efeitos foram avaliados pelo período de exposição (24, 48 e 72 horas).

Os dados foram submetidos à análise de variância e as médias dos dados qualitativos foram comparadas pelo teste de Skott-Knott a 5\% de probabilidade para os tratamentos. Os dados quantitativos foram submetidos à análise de regressão e as variáveis resposta foram analisadas pela correlação de variáveis. Para avaliar o efeito dos períodos de exposição dos ácaros aos extratos vegetais foi adotada a análise multivariada por rede de correlações. As análises estatísticas e a confecção dos gráficos foram realizadas no software R versão 3.6.1 (R Development Core Team, 2019).

\section{Resultados e Discussão}

De acordo com teste $\mathrm{F}$ foi possível organizar os tratamentos no esquema fatorial $(\mathrm{p}<$ 0,01) para avaliar a mortalidade dos ácaros no período de 48 horas de exposição aos extratos vegetais (M48). Porém, a mortalidade dos demais períodos de exposição (M24 e M72) foi influenciada significativamente apenas pelas concentrações dos extratos vegetais utilizados. Essas duas variáveis foram fortemente influenciadas $(\mathrm{P}<0,01)$ pelo fator concentração (Tabela 1) e foi possível comparar as concentrações dos substratos pelo teste de regressão.

Tabela 1. Resumo da Análise de Variância com os valores dos quadrados médios e a significância do teste F para mortalidade (M) do Polyphagotarsonemus latus em diferentes periodos de exposição (24, 48 e 72 h) aos extratos vegetais.

\begin{tabular}{ccccc}
\hline F.V & G.L & M24 & M48 & M72 \\
\hline Extrato vegetal (E) & 2 & $1,6213^{\mathrm{ns}}$ & $0,954^{\mathrm{ns}}$ & $0,561^{\mathrm{ns}}$ \\
Concentração (C) & 4 & $11,6172^{* *}$ & $33,854^{* *}$ & $58,815^{* *}$ \\
E * C & 8 & $0,5984^{\mathrm{ns}}$ & $3,195^{* *}$ & $1,056^{\mathrm{ns}}$
\end{tabular}

F.V: Fontes de variação, G.L: Graus de liberdade, ${ }^{\text {ns }} \mathrm{e}^{* *}$ não significativo e significativo a $1 \%$ pelo teste F. Valores foram transformados em $(x+1)^{1 / 2}$.

Para as variáveis M24 e M72 não foi encontrada diferença significativa $(P>0,05)$ tanto no efeito isolado quanto na interação entre os fatores, para os tipos de extratos vegetais. Já para concentração do extrato em todos os casos foi obtido efeito significativo $(\mathrm{P}<0,05)$ para esse fator isolado, sendo que para M48 também foi encontrada significância na interação entre os fatores. $\mathrm{Na}$ análise de regressão foi obtida significância $(\mathrm{P}<0,05)$ para os modelos 
linear e quadrático, sendo os desvios de regressão não significativos para todas as variáveis testadas (M24, M48 e M72), demonstrando bom ajuste dos dados. O modelo quadrático foi escolhido por apresentar maior coeficiente de determinação, superiores a 70\% em todos os casos (Figuras 1, 2 e 3).

De acordo com a equação de regressão, independente da fonte vegetal, observa-se que os dados de M24 (Figura 1a) e M72 (Figura 1b) apresentaram comportamento quadrático, com 97\% e 79\% dos dados ajustados, respectivamente. Sendo o aumento da mortalidade influenciado pela concentração utilizada.

Figura 1. Curva do ajuste da equação para mortalidade nos períodos (a) de 24 h (M24) e (b) 72 h (M72) em função de exposição às concentrações dos extratos vegetais.

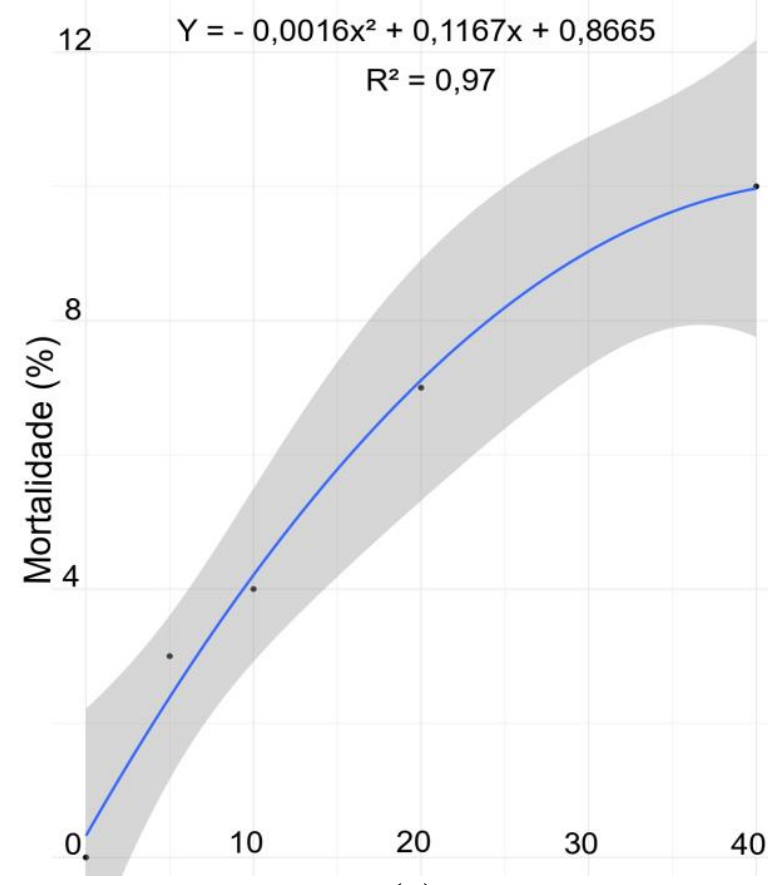

(a)

Fonte: Autores.

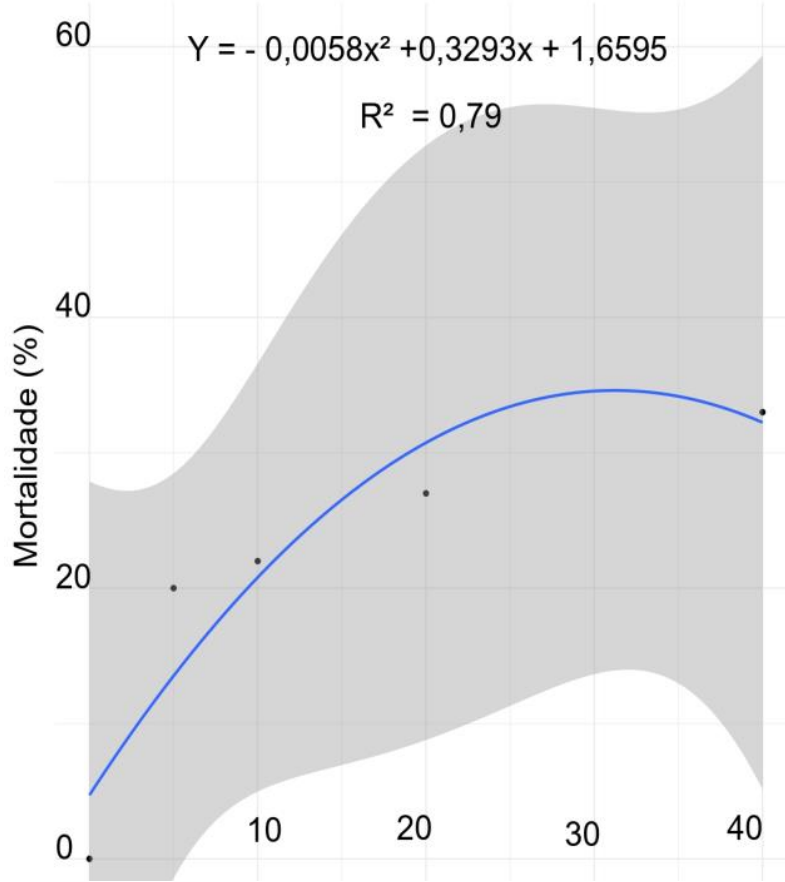

(b)

Através da interação dos efeitos quantitativos e qualitativos, foi possível plotar três gráficos para a variável M48, permitindo estudar o efeito das concentrações por extratos vegetais. Dessa forma, $92 \%$ e $77 \%$ dos dados ajustaram para o modelo quadrático com o uso das concentrações do extrato de Cedrela fissilis (Figura 1b) e Melia azedarach (Figura 2a), respectivamente; e $95 \%$ dos dados são explicados pelo modelo quadrático para as concentrações de extrato de Azadirachta indica (Figura 2b). 
(CC BY 4.0) | ISSN 2525-3409 | DOI: http://dx.doi.org/10.33448/rsd-v9i8.5455

Figura 2. Curva do ajuste da equação para mortalidade no período de $48 \mathrm{~h}$ em função de exposição (a) às concentrações do extrato de Melia azedarach e (b) às concentrações do extrato de Azadirachta indica e (c) Cedrela fissilis.

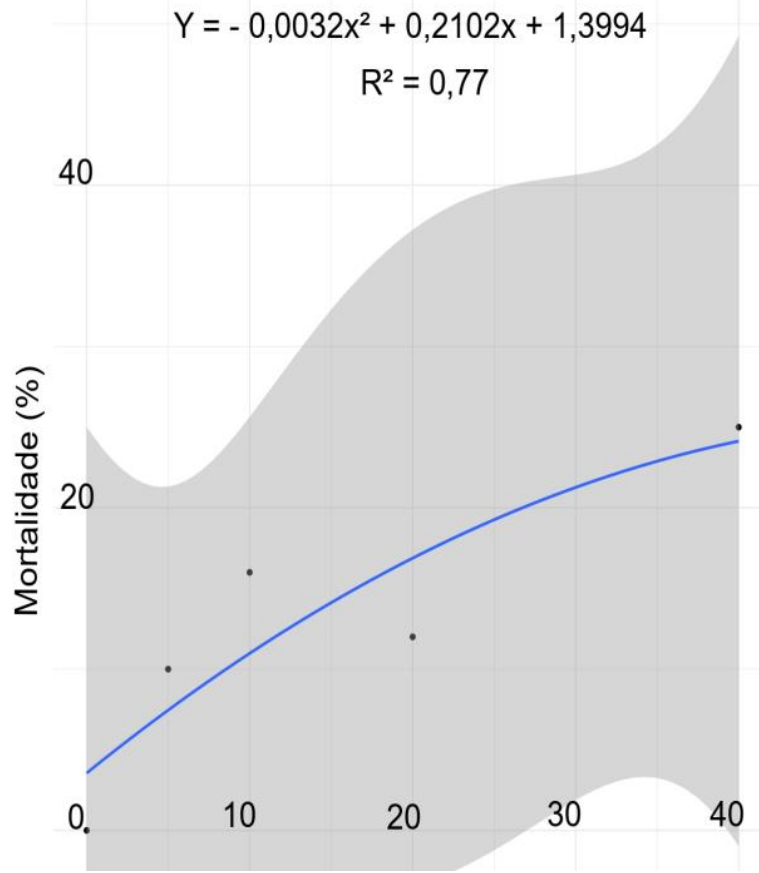

(a)

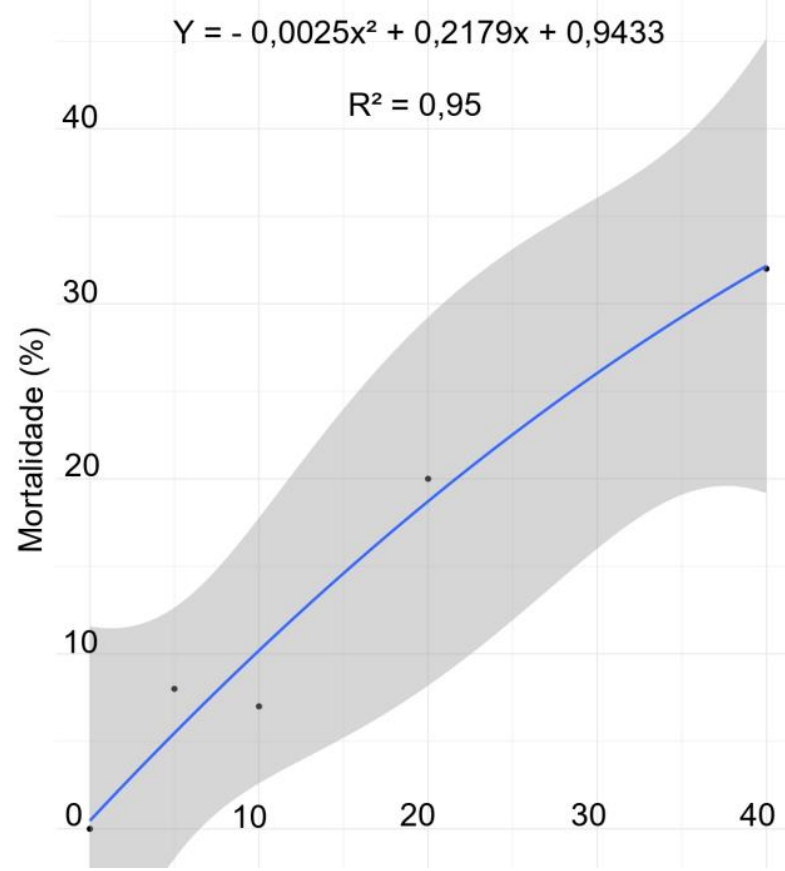

(b)

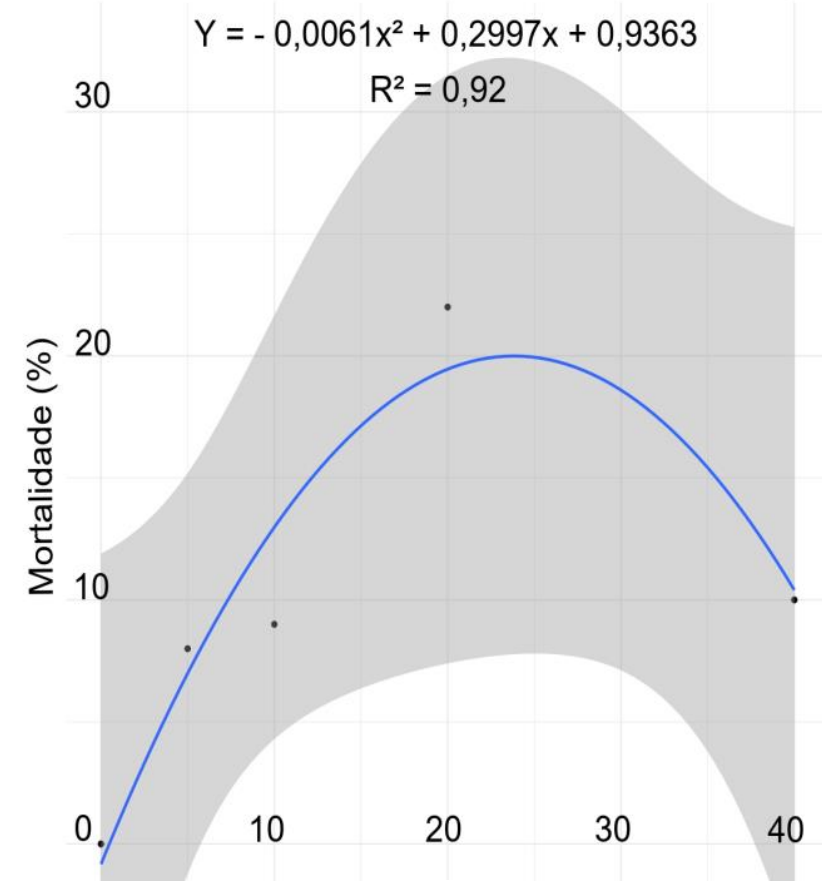

(c)

Fonte: Autores. 
Enquanto que o efeito das concentrações dentro do fator Extrato foi demonstrado com os testes de regressão, para o estudo do fator Concentração e a comparação do desempenho dos diferentes extratos foi necessário aplicar o teste de média (Tukey, $\mathrm{P}<0,05$ ). M24 e M72 não apresentaram diferença significativa (Tabela 1) e suas médias gerais foram de 4,87 e 20,6, respectivamente. No entanto, M48 apresentou diferença significativa para os extratos vegetais (Tabela 1): dessa maneira, dentro das concentrações 0,5 e $20 \%$ não houve diferença significativa entre os extratos vegetais utilizados; na concentração de $10 \%$ a menor média observada foi obtida com o uso do extrato de Azadirachta indica. Na concentração de $40 \%$, os extratos de M. Azedarach e A. Indica apresentaram maior M48 (Tabela 2).

Tabela 2. Efeito dos extratos vegetais na mortalidades de P. latus $48 \mathrm{~h}$ de exposição (M48).

\begin{tabular}{cccc}
\hline Concentração $(\%)$ & Cedro (C. fissilis) ${ }^{*}$ & Cinamomo $(\text { M. azedarach })^{*}$ & Nim $(\text { A. indica })^{*}$ \\
\hline $0^{\mathrm{ns}}$ & $0 \mathrm{a}$ & $0 \mathrm{a}$ & $0 \mathrm{a}$ \\
$5^{\mathrm{ns}}$ & $8 \mathrm{a}$ & $10 \mathrm{a}$ & $8 \mathrm{a}$ \\
$10^{*}$ & $9 \mathrm{ab}$ & $16 \mathrm{a}$ & $7 \mathrm{~b}$ \\
$20^{\mathrm{ns}}$ & $22 \mathrm{a}$ & $12 \mathrm{a}$ & $20 \mathrm{a}$ \\
$40^{*}$ & $10 \mathrm{~b}$ & $25 \mathrm{a}$ & $32 \mathrm{a}$ \\
\hline
\end{tabular}

${ }^{\text {ns }}$ e * não significativo e significativo a $5 \%$ pelo teste $\mathrm{F}$. Médias seguidas pela mesma letra na linha não difere entre si pelo teste Tukey a 5\%.

Na rede de correlação, os valores dos graus de correlação maior que $70 \%$ ( $r>0,7$ ) foram considerados significativos e graficamente representados por bordas mais espessas. Nesse estudo todas as correlações foram positivas. Nesse sentido, a correlação mais expressiva foi obtida pelos pares M72 e M48 $(r=0,77)$ e a menos expressiva obtida entre M24 e M48 ( $r=0,53)$ assim como M24 e M48 ( $r=0,6)$. Dessa forma, o tempo de exposição menos significativo foi o período de 24 horas (Figura 3 ).

Quanto ao efeito dos diferentes extratos vegetais utilizados, nota-se que os extratos de Melia azedarach e Azadirachta indica apresentaram maior eficiência na mortalidade de $P$. latus expostos no perído de 48 horas. De acordo com Veronez et al. (2012), o extrato de Melia azedarach teve ação seletiva quando em estudo com o Tetranychus urticae, fitófago cosmopolita e seu predador, utilizável em controle biológico, o Phytoseiulus macropilis: causando elevada mortalidade na população do ácaro fitófago e não tendo efeito na mortalidade de seu predador. 
Figura 3. Rede de correlações entre mortalidade de $P$. latus sob efeito de diferentes concentrações de extratos vegetais e diferentes períodos: 24h (M24), 48h (M48) e 72h (M72).

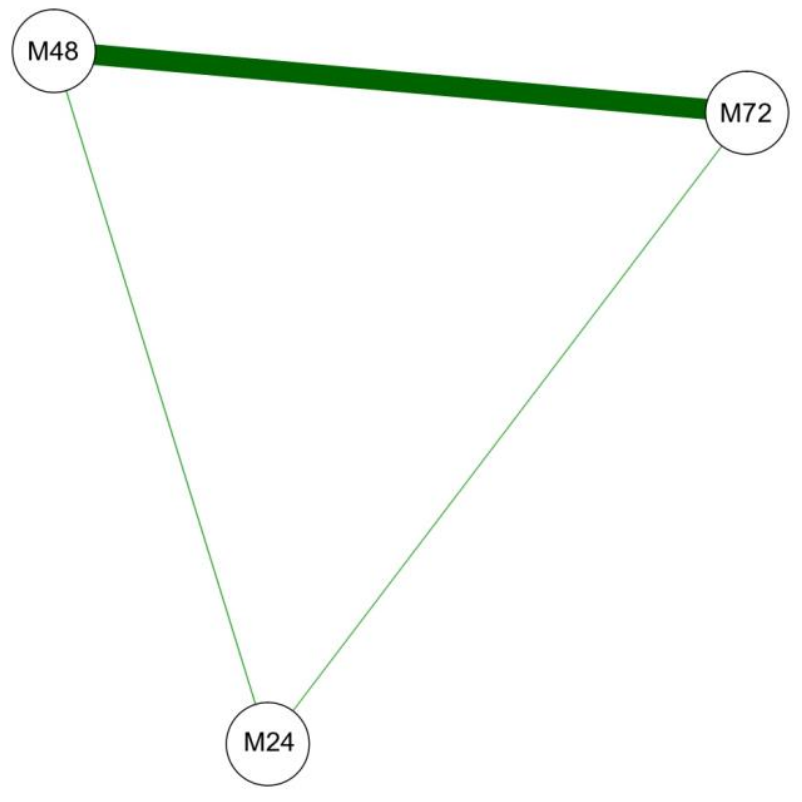

Fonte: Autores.

De acordo com Schmutterer (1990) a azadiractina é o principal composto bioativo quantitativamente isolado de sementes de Azadirachta indica, e outras Meliaceaes. A azadiractina está associada à ação supressora de apetite ou inibidora de crescimento em insetos (Viegas Júnior, 2003). Análises de microscopia, em Rhipicephalus sanguineus expostos às moléculas de azadiractina de fontes vegetais, demonstraram uma diminuição da espessura da cutícula e da espessura das dobras tegumentares com o aumento da dose de azadiractina. Seus efeitos alteraram, principalmente, a morfologia do sistema digestório e reprodutivo de alguns artrópodes dificultando a sobrevivência da população, incapacitando-os de atingir estádios maduros e, consequentemente, produzir novos indivíduos (Souza, et al., 2017).

Veronez et al. (2012) ao comparar a toxicidade de compostos orgânicos com sintéticos, com moléculas ativas semelhantes, relatam que os extratos vegetais podem apresentar uma variação de quantidade de compostos ativos por causa do histórico de manejo até a época de coleta dos materiais vegetais. Tendo em vista que esses compostos com funções ativas sobre a mortalidade e/ou repelência ao organismo alvo são moléculas do metabolismo secundário vegetal influencia no controle de ácaros, já que em seu trabalho foi verificado que extratos aquosos de cinamomo na concentração de $10 \%$ possuiu baixa eficiência no controle de T. urticae. Além da época de coleta dos materiais vegetais 
influenciar a composição bioquímica dos extratos (Moraes, 2009), os metabólitos secundários se encontram distribuídos de modo desigual nos orgãos vegetais (Gonçalves, et al., 2001) sendo um motivo de mais estudos.

As concentrações dos extratos vegetais apresentaram maior influência na mortalidade dos ácaros (Tabela 1) e o tempo de exposição de 48 e 72 horas foram mais expressivos, de acordo com a rede de correlações das variáveis, para o efeito dos extratos (Figura 6). Nesse tipo de análise estatística as correlações entre as variáveis podem ser positivas, neutras ou negativas. Na rede obtida no presente trabalho, a associação dos períodos de exposição com a mortalidade dos ácaros foram todas positivas. No entanto, essa análise tem sido utilizada em estudos agronômicos para determinar, dentre todas as variáveis, as de maior importância no estudo (Teodoro, et al., 2018).

No presente ensaio o maior valor de mortalidade (33\%) foi obtido de P. Latus. Mais estudos para demonstrar os efeitos acaricidas desses óleos administrados em doses e períodos de exposição maiores são recomendados.

\section{Considerações Finais}

Os extratos vegetais apresentaram efeito acaricida promovendo a mortalidade de $P$. Latus. Os extratos vegetais apresentaram maior efetividade nos períodos de 48 e 72 horas. No período de exposição de 48 horas recomenda-se as concentrações e respectivas fontes de extratos vegetal: $24,57 \%$ de Cedrela fissilis, 32,84\% de Melia azedarach ou 43,58\% de Azadirachta indica. Para a exposição de 72 horas, é recomendado 28,39\% de qualquer um dos três extratos vegetais.

\section{Referências}

Alvarenga, A. P. \& Carmo, C. A. F. S. (2014). Seringueira. 2ª ed. Viçosa: EPAMIG, 1056 p.

Castiglioni, E., Vendramim, J. D. \& Tamai, M. A. (2002). Evaluación del efecto tóxico de extractos acuosus y derivados de meliáceas sobre Tetranychus urticae (Koch) (Acari: Tetranychidae). Agrociencia, 6 (2), 75-82. 
Champagne, D. E., Koul, O., Isman, M. B., Scudder, G. G. E. \& Towers, G. H. N. (1992).

Biological activity of limonoids from the Rutales. Phytochemistry, 31, 377-394, doi: 10.1016/0031-9422(92)90003-9

Chaves, P. P., Ruokolainen, K. \& Tuomisto, H. (2018). Using remote sensing to model tree species distribution in Peruvian lowland Amazonia. Biotropica, 50 (5), 758-767. doi:10.1111/btp.12597

Feres, R. J. F. (2000). Levantamento e observações naturalísticas da acarofauna (Acari, Arachnida) de seringueiras cultivadas (Hevea spp., Euphorbiaceae) no Brasil. Revista Brasileira de Zoologia, 17 (1), 157-173.

Gallo, D., Nakano, O., Silveira Neto, S., Carvalho, R. P. L., Baptista, G. C., Berti Filho, E., Parra, J. R. P., Zucchi, R. A., Alves, S. B., Vendramim, J. D., Marchini, L. C., Lopes, J. R. S. \& Omoto, C. (2002). Entomologia agrícola. Piracicaba: FEALQ.

Gonçalves, M. E. C., Oliveira, J. V., Barros, R., Torres, J. B. (2001). Efeito de extratos vegetais sobre estágios imaturos e fêmeas adultas de Mononychellus tanajoa (Bondar) (Acari: Tetranychidae). Neotropical entomology, 30 (2), 305-309, doi: 10.1590/S1519566X2001000200015

Gonçalves, R. \& Vendramim, J. D. (2004). Efeito de Extratos de Meliáceas Sobre o Parasitóide de Ovos Trichogramma pretiosum Riley (Hymenoptera: Trichogrammatidae). Neotropical Entomology, v.33, n.5, p.607-612, doi: 10.1590/S1519-566X2004000500010

Halfeld-Vieira, B. A., Marinho-Prado, J. S., Nechet, K. L., Morandi, M. A. B. \& Bettiol, W. (2016). Defensivos Agrícolas Naturais: uso e perspectivas. Brasilia: EMBRAPA.

Liu, S., Lan, J., Zhou, B., Qin, Y., Zhou, Y., Xiao, X., Yang, J., Gou, J., Qi, J., Huang, Y. \& Tang, C. (2015). HbNIN2, a cytosolic alkaline/neutral-invertase, is responsible for sucrose catabolism in rubber-producing laticifers of Hevea brasiliensis (para rubber tree). New Phytologist, 206 (2), 709-725. doi: 10.1111/nph.13257 
Lorenzi, H. (2000). Árvores brasileiras: manual de identificação e cultivo de plantas arbóreas nativas do Brasil. Nova Odessa: Ed. Plantarum.

Melo, T. M. P., Silva, E. M., Silva, A. G., Vieira, G. H. \& Lopes, B. G. (2019). Syzygium aromaticum Essential Oil to the Control of Tenuipalpus heveae. Journal of Agricultural Science, 11 (8), 295 - 301. doi:10.5539/jas.v11n8p295

Moraes, GJ. \& Flechtmann, CHW. (2008). Manual de acarologia: acarologia básica e ácaros de plantas cultivadas no Brasil. Ribeirão Preto: Holos.

Morais, L. A. S. (2009). Influência dos fatores abióticos na composição química dos óleos essenciais. Horticultura Brasileira, 27, 4050-4063.

Nardo, E. A. B., Costa. A. S. \& Lourenção, A. L. (1997). Melia azedarach extract as na antifeedant to Bemisa tabaci (Homoptera: Aleyrodidae). Florida Entomologist, v. 80, p.92-94. doi:10.2307/3495981.

Nicastro, R. L., Sato, M. E. \& Silva, M. Z. (2010). Milbemectin resistance in Tetranychus urticae (Acari: Tetranychidae): selection, stability and cross-resistance to abamectin. Experimental and Applied Acarology, 50, 231-241.

Oliveira, M. S. S., Railda, A., Arruda, E. J., Marques, A. S. (2007). Eficiência de produtos vegetais no controle da lagarta-do-cartucho-do-milho Spodoptera frugiperda (J.E.Smith, 1797) (Lepidoptera: Noctuidae). Ciência e Agrotecnologia, 31 (2), 326-331. doi: 10.1590/S1413-70542007000200009

Pinto, Z. T., Carneiro, J. F., Carriço, C., Caetano, R. L., Ferreira, V. S. B., Mendonça, P. M., Berenger, A. L. R. \& Figueiredo, MR. (2018). Acaricidal effects of seven Brazilian plant extracts. Revista Colombiana de Entomolología, 44 (1), 44-47. doi: 10.25100/socolen.v44i1.6541

R Development Core Team. (2019). R language definition. Disponível em: https://cran.rproject.org/doc/manuals/r-release/R-lang.pdf. 
Roel, A. R. (2001). Utilização de plantas com propriedades inseticidas: uma contribuição para o desenvolvimento rural sustentável. Revista Internacional de Desenvolvimento Local, 1 (2), 43-50.

Schmutterer, H. (1990). Properties and potential of natural pesticides from the neem tree, Azadirachta indica. Annual Reviews of Entomology, 35, 271-297.

Schuster, M. Z., Rohde, C., Broetto, D. \& Szymczak, L. S. (2009). Efeito Inseticida de Extrato Aquoso de Cinamomo e Macela em Pulgão Aphis gossypii (Hemiptera: Aphididae) em Pepino. Revista Brasileira de Agroecologia, 4 (2).

Silva, R. S. M., Toscano, L. C., Silva, E. M., Andrade, J. R. \& Merlotto, G. R. (2018). Extratos Hidroalcóolicos de Annona squamosa L. e Annona muricata L. (Anonnaceae) na Mortalidade de Pulgões da Família Aphididae em Mostarda. Cadernos de Agroecologia, 13 (2).

Soriosseiri, A., Moshaverinia, A., Khodaparast, MHH. \& Kalidari, G. A. (2018). In vitro acaricidal effect of Melia azedarach ripe fruit extract against Dermanyssus gallinae (Acari: Dermanyssidae). Persian Journal of Acarology, 7 (2), 203-208 doi: 10.22073/pja.v7i2.35065

Souza, J. R. L., Remedio, R. N., Arnosti, A., Abreu, R. M. M. \& Camargo-Mathias, MI. (2017). The effects of neem oil (Azadirachta indica A. JUSS) enriched with different concentrations of azadirachtin on the integument of semi-engorged Rhipicephalus sanguineus sensu lato (Acari: Ixodidae) females. Microscopy Research and Technique, v. 80, n. 8, 838844. doi:10.1002/jemt. 22871

Teodoro, P. E., Carvalho, L. P., Rodrigues, J. I. I., Farias, F. J. C., Carneiro, P. C. S. \& Bhering, L. L. (2018). Interrelations between agronomic and technological fiber traits in upland cotton. Acta Scientiarum. Agronomy, 40, e39364, doi: 10.4025/actasciagron.v40i1.39364

Souza, J. R. L., Remedio, R. N., Arnosti, A., Abreu, A. M. M. \& Camargo-Mathias, MI. (2017). The effects of neem oil (Azadirachta indica A. JUSS) enriched with different concentrations of azadirachtin on the integument of semi-engorged Rhipicephalus sanguineus 
sensu lato (Acari: Ixodidae) females. Microscopy Research and Technique, v. 80, p. 838-844. doi: $10.1002 /$ jemt.22871

Veronez, B., Sato, M. E. \& Nicastro, R. L. (2012). Toxicidade de compostos sintéticos e naturais sobre Tetranychus urticae e o predador Phytoseiulus macropilis. Pesquisa Agropecuária Brasileira, 47 (4), 511-518, doi: 10.1590/S0100-204X2012000400006

Viegas Júnior, C. (2003). Terpenos com atividade inseticida: uma alternativa para o controle químico de insetos. Química Nova, 26 (3), 390-400, doi: 10.1590/S010040422003000300017

Yessinou, R. E., Akpo, Y., Adoligbe, C., Adoligbe, C., Adinci, J., Assogba, M. N., Koutinhouin, B., Karim, I. Y. A. \& Farougou, S. (2016). Resistance of tick Rhipicephalus microplus to acaricides and control strategies. Journal of Entomology and Zoology Studies, 4 (6), 408-414.

\section{Percentage of contribution of each author in the manuscript}

Abimael Gomes da Silva $-25 \%$

Gustavo Haralampidou da Costa Vieira - 25\%

Thalia Mayara Pereira de Melo - 20\%

Glaucia Amorim Faria - 15\%

Eliamara Marques da Silva - 15\% 